\title{
Conductivity mechanism in ionic 2D carbon nitrides: from hydrated ion motion to enhanced photocatalysis
}

Julia Kröger, ${ }^{\text {a, b, c }}$ Filip Podjaski, ${ }^{\text {a c }}$ Gökcen Savasci, ${ }^{\text {a, b, c }}$ Igor Moudrakovski, ${ }^{a}$ Alberto Jimenez-Solano, ${ }^{\text {a }}$ Maxwell W. Terban, ${ }^{\mathrm{a}}$ Sebastian Bette, ${ }^{\mathrm{a}}$ Viola Duppel, ${ }^{\mathrm{a}}$ Markus Joos, ${ }^{\mathrm{a}}$ Alessandro Senocrate, ${ }^{\mathrm{a}}$ Robert Dinnebier, ${ }^{\text {a }}$ Christian Ochsenfeld, ${ }^{\text {a, b, c }}$ Bettina V. Lotsch $*$ a, b, c

${ }^{a}$ Max Planck Institute for Solid State Research, Heisenbergstr. 1, 70569 Stuttgart, Germany

E-mail: b.lotsch@fkf.mpg.de

${ }^{\mathrm{b}}$ Department of Chemistry, University of Munich, LMU, Butenandtstr. 5-13, 81377 Munich, Germany

${ }^{c}$ Cluster of excellence e-conversion, Lichtenbergstr. 4a, 85748 Garching, Germany

Carbon nitrides are among the most studied materials for photocatalysis, however, limitations arise from inefficient charge separation and transport within the material. Here, this aspect is addressed in the $2 \mathrm{D}$ carbon nitride poly(heptazine imide) (PHI) by investigating the influence of various counterions, such as $M=\mathrm{Li}^{+}, \mathrm{Na}^{+}, \mathrm{K}^{+}, \mathrm{Cs}^{+}, \mathrm{Ba}^{2+}, \mathrm{NH}_{4}{ }^{+}$and tetramethyl ammonium, on the material's conductivity and photocatalytic activity. These ions in the PHI pores affect the stacking of the 2D layers, which further influences the predominantly ionic conductivity in $M$-PHI. Nacontaining PHI outperforms the other $M$-PHI in various relative humidity $(\mathrm{RH})$ environments $(0$ $42 \% \mathrm{RH}$ ) in terms of conductivity, likely due to pore channel geometry and size of the (hydrated) ion. With increasing RH, the ionic conductivity increases by 4-5 orders of magnitude (for Na-PHI up to $10^{-5} \mathrm{~S} \mathrm{~cm}^{-1}$ at $\left.42 \% \mathrm{RH}\right)$. At the same time, the highest photocatalytic hydrogen evolution rate is observed for Na-PHI, which is mirrored by increased photo-generated charge carrier lifetimes, pointing to efficient charge carrier stabilization by mobile ions. These results indicate that ionic conductivity is an important parameter that can influence the photocatalytic activity. Besides, RHdependent ionic conductivity is of high interest for separators, membranes, or sensors.

Key words: Carbon nitrides, mixed conductors, humidity dependent ion conduction, photocatalysis, poly(heptazine imide) 


\section{Introduction}

Our energy-intensive society has an increasing demand for alternative, renewable energy sources due to their natural abundance and increasing environmental pollution by the current predominantly used energy sources. ${ }^{[1]}$ Consequently, solar-driven energy conversion technologies, including photocatalytic water splitting, are coming to the fore, with an increasing focus on earthabundant organic materials. Among the wide variety of photocatalysts, carbon nitride polymers are among the most widely studied classes of semiconductor materials due to their affordability and ease of synthesis, chemical and thermal stability, suitably positioned visible light band gap, and high activity. Since the first report in 2009 by Wang et al. ${ }^{[2]}$ on the photocatalytic activity of 1D melon-type carbon nitride for water splitting, the number of reports on modified carbon nitrides with increasing performance has grown tremendously. ${ }^{[3]}$

In recent years, a novel carbon nitride called poly(heptazine imide) (PHI) was identified, bearing an intriguing dual functionality of light absorption and simultaneous charge storage. ${ }^{[4-6]}$ This $2 \mathrm{D}$ polymer is built up of heptazine units forming pores, which can be filled with hydrated alkali ions, in contrast to the archetype carbon nitride melon, often simplistically called $\mathrm{g}-\mathrm{C}_{3} \mathrm{~N}_{4}$ in the literature. ${ }^{[4]}$ PHI's intrinsic energy storing ability have recently enabled hybrid concepts interfacing energy conversion and storage, such as "dark photocatalysis". Akin to natural photosynthesis it temporally decouples the process of light absorption from the catalytic conversion (or fixation) of light-induced charge carriers, which instead is triggered in the dark. ${ }^{[5]}$ By illuminating the yellow PHI suspension in the presence of an electron donor, a photo-reduced blue state is generated, which is stable for several days in the absence of oxygen. ${ }^{[4,5]}$ Then, a cocatalyst like Pt can be added, and solar hydrogen is produced on demand in the absence of light. Photocatalytic propulsion of PHI Janus particles or a direct electric discharge of the photo-reduced, blue state is also possible, enabeling solar batteries to be realized from this abundant material. ${ }^{[6,7]}$ However, the long-term charge storage process itself can limit the direct photocatalytic activity by enhancing parasitic recombination of the light-induced charge carriers as charge builds up on the system. ${ }^{[8]}$ An in-depth study of charge storage mechanism is still missing while being of high interest for the targeted design of (photo)electrochemical energy conversion and storage. ${ }^{[6,9]}$

Besides charge storage, charge carrier transport is a crucial parameter, not only in electrical energy storing devices but also in photocatalysis. ${ }^{[10]}$ The overall photocatalytic performance of a particular 
system is the sum of various processes that occur at different timescales; from below ps to a few seconds, which in principle can all be limiting, but also individually optimized to obtain more efficient systems. ${ }^{[1,12]}$ These processes include light absorption, charge carrier separation, their transport to the solid-liquid interface and to the surface catalytic center or the co-catalyst, where catalytic reactions take place. At the moment, mobility and diffusion of charge carriers in carbon nitrides is not well understood. The pertinent picture is that of predominantly localized charge carriers with low mobility. Insights into charge carrier mobility and diffusion can thus provide valuable information on carrier lifetimes, diffusion lengths, electronic conductivity, activation energies for diffusion, and transfer coefficients. ${ }^{[11,13]}$ In addition, it is not clear whether an intrinsic, enhanced ionic or electronic conductivity can be beneficial for lifetimes of photo-generated charges, which would increase the photocatalysis rate.

Carbon nitrides are generally considered polymeric semiconductors that behave like "quasi monomers" ${ }^{[14]}$ with largely localized charge carriers and low or local intrinsic conductivity. For example, Merschjann et al. ${ }^{[15]}$ characterized the charge transfer (of excitons as dominant charge carriers) in the $1 \mathrm{D}$ carbon nitride melon as an interlayer conduction mechanism by transient absorption spectroscopy (TAS). It was observed that the exciton dissociates into a singlet polaron pair, which then diffuses out-of-plane via overlapping molecular orbitals. Such transport is electronically beneficial in contrast to an electron transfer within the polymer chain, due to a high energy barrier caused by imide bridges, which interrupts in-plane conjugation. ${ }^{[15,16]}$ Out-of-plane charge hopping was recently also postulated in the 2D carbon nitride poly(triazine imide) (PTI) by TAS and electrochemical measurements. Noda et al. ${ }^{[17]}$ reported an anisotropic charge transport behavior, where the out-of-plane overall conductivity $\sigma\left(1.0 \times 10^{-4} \mathrm{~S} \mathrm{~cm}^{-1}\right)$ was reported to be two orders of magnitude greater than the in-plane conductivity $\left(1.5 \times 10^{-6} \mathrm{~S} \mathrm{~cm}^{-1}\right)$. However, no distinction between the ionic $\left(\sigma_{\text {ion }}\right)$ and electronic conductivity $\left(\sigma_{\text {el }}\right)$ was reported, while it is likely that ions significantly contribute to the overall conductivity, since lithium ion motion in porous PTI was already reported for PTI-based cathode materials. ${ }^{[18]}$ Besides, proton transport via a Grotthuss mechanism was suggested for PTI in humid environments. ${ }^{[19]}$ With increasing humidity the conductivity was found to be enhanced by orders of magnitude, ${ }^{[20,21]}$ which can be exploited in devices like field effect transistors ${ }^{[22]}$ or actuators. ${ }^{[9,23]}$ 
So far, little information is available on the dominant type of charge carrier transport (ionic $\sigma_{\text {ion }}$ or electronic $\sigma_{\mathrm{el}}$ ) for carbon nitrides, or the influence of conductivity on the photocatalytic performance. Since PHI has the unique ability to store long-lived electrons, we have chosen this carbon nitride as a model system to study its conductivity mechanism. Depending on the cation being present in the structural pores, we find that the stacking of the 2D PHI polymer is altered by the effective size of the hydrated cation and its interaction strength with the polymer backbone. This further influences the channel geometry and the ion motion within those channels. Cation containing PHI (M-PHI), a mixed conductor, has an at least two orders of magnitude higher ion conductivity as compared to the electron conductivity. Due to an interplay of optimized geometry, effective ionic radius, and backbone interaction, dry Na-PHI exhibits an order of magnitude better conductivity than K-PHI, followed by $\mathrm{Li}^{+} \sim \mathrm{Cs}^{+} \sim \mathrm{NH}_{4}{ }^{+}>$tetramethyl ammonium $\left(\mathrm{TMA}^{+}\right)>>\mathrm{Ba}^{2+}$ containing PHI materials. This trend remains when increasing the relative humidity (RH) from dry conditions to ambient $\mathrm{RH}$ of approximately $42 \%$, where all materials show an increase in conductivity of 4-5 orders of magnitude and are almost fully hydrated. In addition, we find that higher ion conduction correlates with a better stabilization of the photo-generated charges under photocatalytic conditions, which is mediated by the mobile counterions in the pores. Likewise, the highest photocatalytic hydrogen evolution rate is found for materials with highly mobile ions as in Na-PHI. Understanding the coupled electronic-ionic processes described herein thus opens new design pathways for rationally enhancing the photocatalytic activity in ion-containing porous materials with tunable optoelectronic and ionic transport properties. ${ }^{[10,24-26]}$

\section{Results and Discussion}

\section{Structural analysis}

Potassium containing poly(heptazine imide) (K-PHI) was synthesized by heating the 1D heptazine polymer melon in a KSCN salt melt as described previously. ${ }^{[27]}$ The resulting ionic $2 \mathrm{D}$ polymer hosts potassium ions in its $1 \mathrm{D}$ pore channels formed along the $c$-direction. ${ }^{[4]}$ Stirring K-PHI in a freshly prepared $1 \mathrm{M} M \mathrm{Cl}_{\mathrm{x}}\left(\mathrm{x}=1,2 ; M=\mathrm{Li}, \mathrm{Na}, \mathrm{K}, \mathrm{Cs}, \mathrm{Ba}\right.$, tetramethyl ammonium (TMA), $\mathrm{NH}_{4}$ ) aqueous solution multiple times results in facile ion exchange $(M-\mathrm{PHI}),{ }^{[28]}$ as evidenced by elemental analysis (Table S1, Figure S1, Supporting information). The concentration of ions present in the PHI pores is not dependent on the type of alkali ion ( $\mathrm{Na}, \mathrm{K}$ or $\mathrm{Li}$ ), but is reduced to 
half when $\mathrm{K}^{+}$is exchanged for $\mathrm{Ba}^{2+}$ ions, consistent with the requirement of overall charge balance. To investigate possible structural changes after ion exchange, the materials were analyzed by a combination of long-range and local structural and spectroscopic techniques, including powder Xray diffraction (PXRD), total scattering pair distribution function (PDF) analysis, transmission electron microscopy (TEM), Fourier-transformed infrared (FT-IR), nuclear magnetic resonance (NMR) and UV-visible light (UV-vis) spectroscopy. Analysis of the stacking order of the 2D PHI layers suggests a structure-directing influence of the incorporated cation, as explained below.

For the as-synthesized material K-PHI, two distinct low angle reflections ( $\overline{1} 10$ and 010 at $8.03^{\circ}$ $2 \theta / 11.0 \AA$ and $9.97^{\circ} 2 \theta / 8.9 \AA$ ) are observed, which are diagnostic of an ordered staggered stacking of the 2D layers (Figure 1a). ${ }^{[4]}$ When the local slipping directions become increasingly disordered, as demonstrated by recursive simulations of the stacking fashion using TOPAS, ${ }^{[29]}$ the two lowangle reflections become more diffuse (Figure S7-S9, Table S3, Supporting information), until both reflections merge, and an additional reflection (120) around $14^{\circ} 2 \theta$ (6.3 $\AA$ ) becomes more pronounced (Figure S8, Supporting information). ${ }^{[4]}$ For small but random layer offsets, the apparent long-range order appears hexagonal, as would be the case for an ordered eclipsed stacking relationship, which is the case for Li-PHI compared to other $M$-PHI materials. Similar behavior is observed in covalent organic frameworks. ${ }^{[30]}$ The water content has a significant impact on the stacking behavior, to an extent that the three-dimensional coherence is strongly decreased if the amount of water is reduced in the pores. ${ }^{[4]}$ The structure of Na-PHI is best described as intermediate to those of K-PHI and Li-PHI.

Lithium ions in an aqueous environment are known to form two hydration shells of 4 to 6 and 7 to 10 water molecules, respectively. In contrast, potassium and sodium ions coordinate only one inner hydration shell with 5 to 6 or 6 to 7 water molecules. ${ }^{[31-33]}$ In agreement with this trend, the water content, which can be removed by heating, detected by thermogravimetric analysis (TGA) for samples under ambient conditions was around 9-10 wt\% (1.5-1.7 $\mathrm{H}_{2} \mathrm{O}$ per heptazine or 2.0-2.3 $\mathrm{H}_{2} \mathrm{O}$ per $\mathrm{K}^{+}$) for K-PHI, 12-13 wt\% (2.0-2.2 $\mathrm{H}_{2} \mathrm{O}$ per heptazine or 2.7-2.9 $\mathrm{H}_{2} \mathrm{O}$ per $\mathrm{Na}^{+}$) for NaPHI, and 14-15 wt\% (2.3-2.4 $\mathrm{H}_{2} \mathrm{O}$ per heptazine or 3.1-3.2 $\mathrm{H}_{2} \mathrm{O}$ per $\mathrm{Li}^{+}$) for Li-PHI (Figure S17, Supporting information). Water retention was measured up to 473-523 K, suggesting that water strongly interacts with the cation or the polymer backbone and indicates that water cannot be removed completely, so that TGA ca only detect a minimum amount of water being present (Figure 
S17, S48, Supporting information). TEM data of K-PHI, Na-PHI (vide infra), and Li-PHI are consistent with the PXRD data, confirming that the 2D backbone remains intact, but the layers adopt different stacking polytypes (Figure 1b-d). The diagnostic first two reflections in PXRD are also visible by TEM, with a constant value around $10.2 \AA$ (110) for all three samples (being slightly smaller than in PXRD), but with decreasing distances of 8.8( \pm 0.1$) \AA$ to $8.3( \pm 0.1) \AA(010)$ on going from K-PHI via Na-PHI to Li-PHI. This TEM observation is in line with signal broadening combined with a slight shift of the maximum of the second reflection to higher angles until merging of the first reflections in PXRD occurs. From these changes, a slightly different pore channel geometry for the different alkali ion containing PHI can be assumed.

Consistent with TEM data, FT-IR (Figure 1e, Figure S14, Supporting information) and NMR spectroscopy (Figure S15, Supporting information) prove that only the stacking is affected by ion exchange, since the structure of the covalent $2 \mathrm{D}$ polymer backbone remains unchanged. ${ }^{[4]}$ The only change observed in direct ${ }^{13} \mathrm{C}$-NMR experiments is a line broadening in Li-PHI compared to K-PHI (Figure S15, Supporting information), whereas the peak position and intensities remain the same. The broadening of the signal at 167-168 ppm, corresponding to carbon atoms next to a primary amine group, points to a slightly larger disorder in the Li-PHI structure, which is consistent with ${ }^{15} \mathrm{~N}-\mathrm{CP}-\mathrm{NMR}$ experiments (Figure S15, Supporting information). ${ }^{[34]}$

These findings are corroborated by PDF analysis. The polymer backbones of K-PHI, Na-PHI, and Li-PHI are shown to be the same due to similarity in sharp peaks assigned to intralayer atom-pair correlations at short distances up to approx. $5 \AA$ (Figure S2-S6, Supporting information). Slight differences in the exact shape and relative intensities in the range above $\sim 3 \AA$ can be affected, for instance, by differences in local structuring of the pore content. Significant additional intensity is present in the range of 2.8 to $3.0 \AA$ for the K-PHI sample, which corresponds to K-O and possibly K-N interactions (Figure 1g, h, Figure S3, Supporting information). The apparent lack of such a correlation for Na-PHI and Li-PHI is explained by the smaller scattering cross-sections of $\mathrm{Na}$ and $\mathrm{Li}$ relative to $\mathrm{K}$. The position is expected to shift due to the smaller distance between the water and $\mathrm{Na} / \mathrm{Li}$ ions as predicted by quantum chemical calculations (Figure S3, S10, S11, S50, Supporting information) - approx. $2.75 \AA$ (K-O), $2.42 \AA$ (Na-O), and $2.0 \AA$ (Li-O). In fact, we do observe subtle increases in diffuse intensity that may correspond to the first $\mathrm{Na}-\mathrm{O}$ and $\mathrm{Li}-\mathrm{O}$ shells, respectively. 
Local structure models, based on that previously determined for highly ordered K-PHI, ${ }^{[4]}$ were fit to the experimental PDFs to further probe for differences in the local stacking behavior (Figure 1g, h, Figure S5, Supporting information). We found that while the interlayer distance in the 001 direction increases from K- $(3.15 \pm 0.01 \AA)$ to Na- $(3.17 \pm 0.01 \AA)$ to Li-PHI $(3.18 \pm 0.02 \AA)$, the on-average neighbor layer offset over the first few interlayer distances appears to decrease (from 2.8 in K-PHI to 2.3 in Li-PHI) (Table S2, Supporting information). This observation also coincides with an increased disorder for both Na-PHI and Li-PHI indicated by broader interlayer stacking correlations. Taken in the context of the apparent hexagonal symmetry of the Li-PHI diffraction pattern, we can conclude the following: $\mathrm{K}^{+}$with their solvation shells drive a larger layer offset, combined with a more unidirectional ordering of the interlayer slipping. This leads to a triclinic lattice, though likely with occasional stacking faults, affecting the pore channel geometry. ${ }^{[4]}$ For Li-PHI, the differences in ion-solvation-layer interactions produce shorter slipping distances that are more directionally disordered, which leads to long-range averaging to a higher apparent symmetry. Less favorable interlayer interactions then likely result in the increased interlayer distances observed for Li-PHI. Na-PHI displays the predominantly K-PHI-type structure but with increased stacking disorder similar to Li-PHI (Figure 1f) (Table S2, S3, Figure S6-S9, Supporting information). ${ }^{[4]}$ Similar to K, Na rather tends to form only one hydration shell, ${ }^{[32,35]}$ however, the radius of hydrated sodium ion (3.6 $\AA$ ) is slightly larger than the one of potassium $(3.3 \AA)$, which might cause the observed increase in layer distance. ${ }^{[36]}$
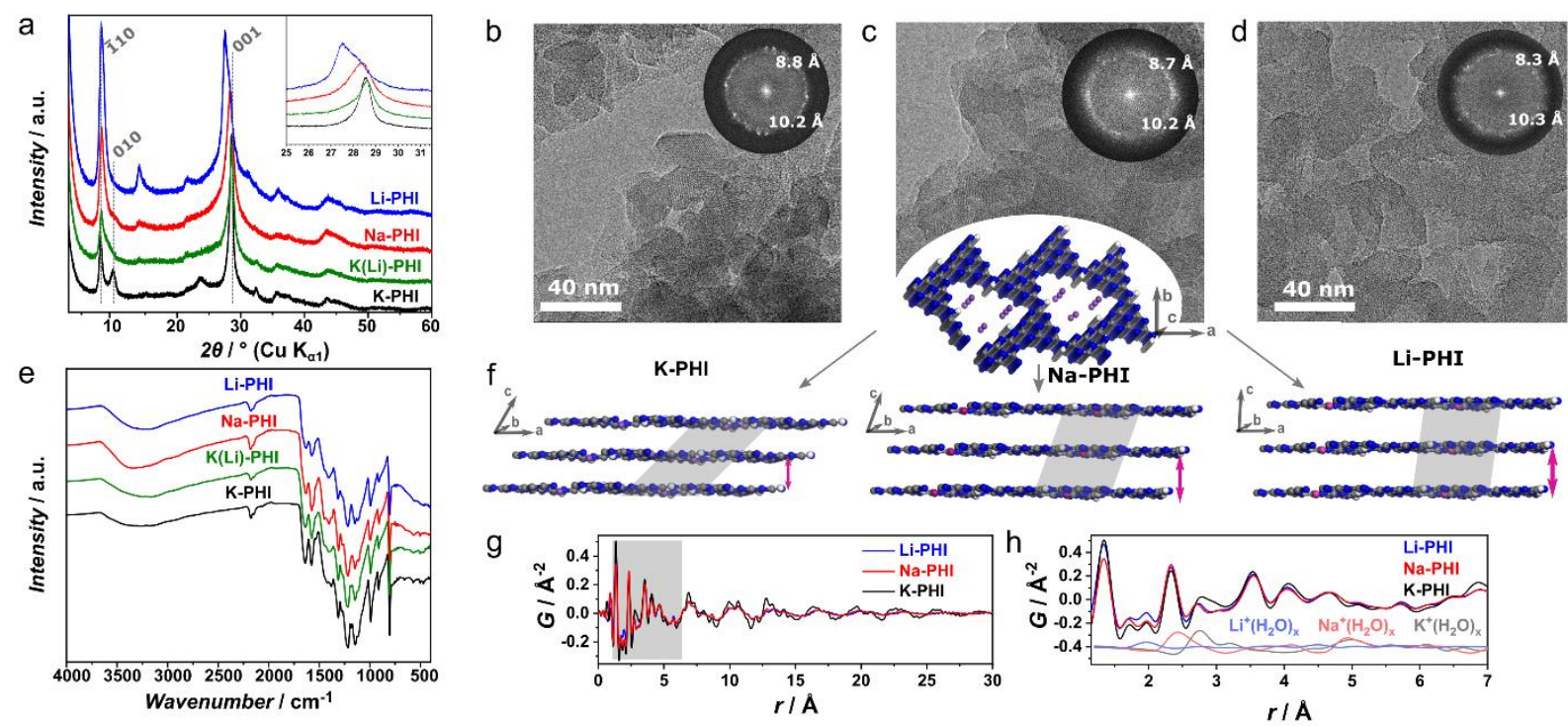

Figure 1: Comparison of PHI filled with different alkali ions in the pores. a) PXRD of K-, Na-, Li-, and K(Li)-PHI; b) TEM image of K-PHI and fast Fourier transformed (FFT) image (inset); c) TEM image of Na-PHI and inset showing fast Fourier transform 
(FFT); d) TEM image of Li-PHI and inset showing fast Fourier transform (FFT); e) FT-IR of K-, Na-, Li-, and K(Li)-PHI; f) Schematic of the idealized difference in stacking order between K-PHI, Na-PHI and Li-PHI and the resulting channels for ion transport (grey shading). g and h) Short- $r$ range of PDFs of Li-, Na- and K-PHI. h) Zoom in the grey area in g). The PDF data for the $\mathrm{K}-/ \mathrm{Na}-/ \mathrm{Li}-$ water shells are obtained from quantum chemical calculations.

The effect of the pore content in the PHI pores, which have a radius of only $3.8 \AA{ }^{[4,6]}$ on the stacking behavior was also tested with even larger ions. Ammonium ions do not affect the structure of PHI compared to K-PHI, most likely a consequence of the similar size of ammonium and potassium ions, both in the presence or absence of water (Table S4, Supporting information). In Cs-PHI, however, a disappearance or significant broadening of the stacking and in-plane reflections in PXRD experiments was observed (Figure S13, Supporting information). This loss might be due to partial dehydration of the $\mathrm{Cs}^{+[37]}$ due to a comparably low hydration energy, ${ }^{[38]}$ or a predicted enhanced interaction energy of carbon nitrides and Cs ions. ${ }^{[39]}$ Similar effects might be present in Ba-PHI, where no stacking reflection is visible any more. Yet, due to the much higher hydration energy of $\mathrm{Ba}^{2+},{ }^{[40]}$ the ion is not likely to be de-hydrated and therefore is too large (4.0 $\AA$ A) to reside in the pore of PHI (Figure S13, Table S4, Supporting information). The same is true for $\mathrm{TMA}^{+}{ }^{[37]}$ where no ion exchange was observed (Table S1, Supporting information). Taking all these findings together, only the stacking behavior and hence the pore channel geometry is affected when exchanging the ions and modifying the water content in the PHI pores. However, only those hydrated ions can be exchanged that fit into the PHI pores, which might hint to a "sweet spot" enabling optimized backbone interactions, pore geometry and transport for a specific hydrated ion size. Especially, when taking into account the calculated minimum spacing for easy sodium ion motion, which was found to be slightly above, the pore diameter of the PHI pore (3.7 А). ${ }^{[41]}$

\section{Conductivity analysis}

As described above, the type of ion residing in the structural pores and the water content significantly influences the stacking of 2D PHI layers. To reveal if those changes have a possible impact on ion mobility and photocatalytic behavior, we first studied the overall conductivity of PHI. ${ }^{[6,28]}$ The mobility of ions in the PHI pores was probed by different techniques spanning various timescales and diffusion distances: Electrochemical impedance spectroscopy (EIS), galvanostatic polarization measurements, and ${ }^{7} \mathrm{Li}$ pulsed field gradient (PFG) NMR were used as long-range sensitive methods, ${ }^{[42-44]}$ whereas NMR line shape analysis and relaxometry were 
employed for correlating the macroscopic ion diffusion. Its microscopic origin is probed by temperature and frequency dependence of the diffusion-induced NMR spin-lattice or spin-spin relaxation rates, which are influenced by local dipolar-magnetic or quadrupolar-electric fluctuations. ${ }^{[42-44]}$

First, the effect of humidity on the conductivity will be discussed, followed by an analysis including different types of ions in the PHI pores. For accurate humidity-dependent electrochemical measurements, the degree of swelling of PHI under humid conditions has to be known prior. Therefore, we analyzed the role of pore water in $M$-PHI by PXRD analysis at RHs between $1 \%$ and $90 \%$, which reveals almost no change in the K-PHI structure with the state of hydration (Figure 2a). The stacking reflection is only shifted from $28.27^{\circ} 2 \theta(3.16 \AA)$ at $1 \% \mathrm{RH}$ to $27.97^{\circ} 2 \theta(3.22 \AA)$ at $90 \% \mathrm{RH}$, indicating that water is not intercalated in-between the layers, but rather within the pore channels, hence not causing swelling. Small changes seen by water uptake are reversible, however, a hysteresis in the wetting - drying behavior can be observed, possibly pointing to a strong interaction of water with the polymer backbone. Also by solid state NMR, no changes could be observed in the PHI structure due to drying (Figure S23, Supporting information).

To conduct EIS and galvanostatic direct current (DC) measurements the $M$-PHI powder was pressed to a pellet with the PHI particles arranging randomly (with approximately $85 \%$ theoretical density) (Table S9, Supporting information). DC and impedance measurements of the various $M$ PHI samples with different applied currents were performed with ion-blocking electrodes since PHI was not stable in a non-blocking configuration (Figure S18-S22, Supporting information). Ionic and electronic conductivity were distinguished in DC measurements by the initial voltage jump of the steady-state response (Figure 2b, Figure S24, Supporting information). ${ }^{[45-48]}$ The DC potential drop over a dried Na-PHI sample is 30 times higher compared to the sample under ambient conditions, i.e., the resistance is increased by a factor of 30 (Figure 2b), similar to the behavior of K- and Li-PHI (Figure S25, Table S6, Supporting information). Additionally, the DC curves for dry samples point to a dominant electric contribution. In contrast, $M$-PHI under ambient conditions at around 30-40 \% RH showed a mixed conduction with a dominantly ionic contribution $\left(10^{-7}-10^{-8} \mathrm{~S} \mathrm{~cm}^{-1}\right)$ to the overall conductivity and a significantly smaller electronic conductivities by two orders of magnitude $\left(10^{-9}-10^{-10} \mathrm{~S} \mathrm{~cm}^{-1}\right)$ (Figure S27, S28, Table S7, Supporting 
information). The electronic conductivity seems not to be affected by the humidity, whereas the ionic conductivity is significantly increasing with enhanced humidity. Even further enhancement in conductivity $\left(10^{-6}-10^{-7} \mathrm{~S} \mathrm{~cm}^{-1}\right)$ can be observed for samples stored in a $75 \% \mathrm{RH}$ environment, clearly pointing to a significant contribution of the water content on the ion conduction.

To validate these results, EIS was measured in a frequency range of $10^{-2}-10^{6} \mathrm{~Hz}$. In general, the Nyquist plots of all PHI samples can be described by one semi-circle at higher frequencies, corresponding to bulk transport processes and a constant phase element (or possibly a second semicircle) at lower frequencies correlated to grain boundary or interface effects (Figure 2c, Figure S29, Supporting information). The high-frequency response was confirmed as a bulk conduction process by extracting the capacitance $\left(10^{-10}-10^{-12} \mathrm{~F}\right)$ and dielectric constant (reaching values of 8-12) supported by PHI particle size-dependent impedance measurements as well as a study with variation of the pellet thickness (Figure S30-S34, Table S8, Supporting information), and might also hint to anisotropic conductivity in this 2D materials. ${ }^{[49]}$ The Nyquist plot of Na-PHI in 2-42 $\% \mathrm{RH}$ is depicted in Figure 2c, showing only one high-frequency semi-circle over the whole humidity range (with a capacitance of $10^{-12} \mathrm{~F}$ ). The overall conductivity, represented by the semicircles, is depending on the water content, in accordance to DC measurements. The conductivity, influenced mainly by the ion motion, for (Na-, K-, Li-)PHI is increased by 4-5 orders of magnitude within this humidity range. However, Na-PHI outperforms the other materials by roughly one order of magnitude under all conditions (e.g. Na-PHI: $4.5( \pm 3.0) \times 10^{-10} \mathrm{~S} \mathrm{~cm}^{-1}$ at $2 \% \mathrm{RH}$ and $2.5( \pm 1.0) \times 10^{-6} \mathrm{~S} \mathrm{~cm}^{-1}$ at $\left.42 \% \mathrm{RH}\right)$ (Figure 2d, Figure S35, Supporting information). Notably, with increasing water content, the dielectric constant of $M$-PHI increases from 5-6 under dry to 8-12 under ambient to 15-16 under humid conditions, as calculated from impedance data (Table S12, Supporting information). This increase suggests a more efficient dielectric screening of the cations by water molecules in response to an electric field, along with additional motional degrees of freedom with increasing pore water content, resulting in higher ion conductivity.

To investigate the effect of different ions on the conductivity, we looked at the conductivity at ambient conditions (around 30-40\% RH), where 9-15 wt\% water is present in PHI, depending on the cation (Figure S17, Supporting information). The highest conductivity values of $5.0( \pm 3.0) \times 10^{-}$ ${ }^{6} \mathrm{~S} \mathrm{~cm}^{-1}$ were obtained for Na-PHI (Li-PHI: $1.0( \pm 1.0) \times 10^{-7} \mathrm{~S} \mathrm{~cm}^{-1}$ and K-PHI: $6.0( \pm 10.0) \times 10^{-8} \mathrm{~S}$ $\mathrm{cm}^{-1}$ ) (Figure 2e with suggested directional conductivity). From these values the diffusion 
coefficients for the ion motion $\left(\mathrm{D}^{\sigma}\right)$ were determined via the Nernst-Einstein equation (Na-PHI: $5.0( \pm 0.7) \times 10^{-14} \mathrm{~m}^{2} \mathrm{~s}^{-1}$, K-PHI and Li-PHI: $\left.5.0( \pm 0.7) \times 10^{-15} \mathrm{~m}^{2} \mathrm{~s}^{-1}\right)$ (Chapter 4.4, Supporting information). The fact that similar values for the activation energy of the three PHI materials were obtained $\left(\mathrm{E}_{\mathrm{a}}=0.46-0.50 \mathrm{eV}\right)$ (Figure 2f, Figure S38, Supporting information) point to similar transport process in the materials and are in the expected range for polymeric systems. ${ }^{[50-52]}$ Under ambient conditions, the cations in the PHI pores are (almost) fully hydrated, which enables motion of the cations in the pores - in contrast to dry conditions, where the de-hydrated cation is strongly interacting with the negatively charged PHI backbone and mainly electronic conductivity is measured with high activation energies (up to $E_{a}=0.84( \pm 0.6) \mathrm{eV}$, Figure S40, Supporting information). ${ }^{[53]}$ When increasing the water content, the transport mechanism of the hydrated cations in the PHI pores is similar for all pore fitting $M$-PHI, as the activation energies under ambient conditions are comparable. However, the overall conductivity values are dependent on the type of cation. This clearly points to a mixed contribution of the hydrated ion size, the stacking behavior of the 2D PHI layers, and hence the pore geometry, which is affected by the type of ion. An optimized hydrated cation size and pore geometry might therefore be fulfilled for Na-PHI, enabling a comparable fast ion conduction. This interpretation is further verified when looking at PHI containing $\mathrm{Cs}^{+}, \mathrm{Ba}^{2+}, \mathrm{NH}_{4}{ }^{+}$or $\mathrm{TMA}^{+}$ions. The conductivity obtained from EIS decreases from Cs- and $\mathrm{NH}_{4}-\mathrm{PHI}$ with similar hydrated ion radii $\left(1.0( \pm 0.5) \times 10^{-7} \mathrm{~S} \mathrm{~cm}^{-1}\right)$ via TMA-PHI with partially remaining $\mathrm{K}^{+}\left(8.0( \pm 9.0) \times 10^{-8} \mathrm{~S} \mathrm{~cm}^{-1}\right)$ to a complete breakdown for Ba-PHI (1.0 $\left.( \pm 0.4) \times 10^{-11} \mathrm{~S} \mathrm{~cm}^{-1}\right)$ (Figure S41, Supporting information). As mentioned in the structure section, $\mathrm{Ba}^{2+}$ with its hydrated ion radius of $4.0 \AA$ is too big (Table S4, Supporting information) to move along the PHI pores, and the energy for dehydration is comparably high, resulting in a low overall conductivity, which might be based on electronic contributions, since the ions are not mobile in this setting. All in all, these findings point to water-assisted ion motion, leading to humidityenhanced conductivity. Such enhanced hydrated ion motion in the $M$-PHI channels in contrast to the non-hydrated ions is affected by the pore channel geometry and the size of the cation being present. 

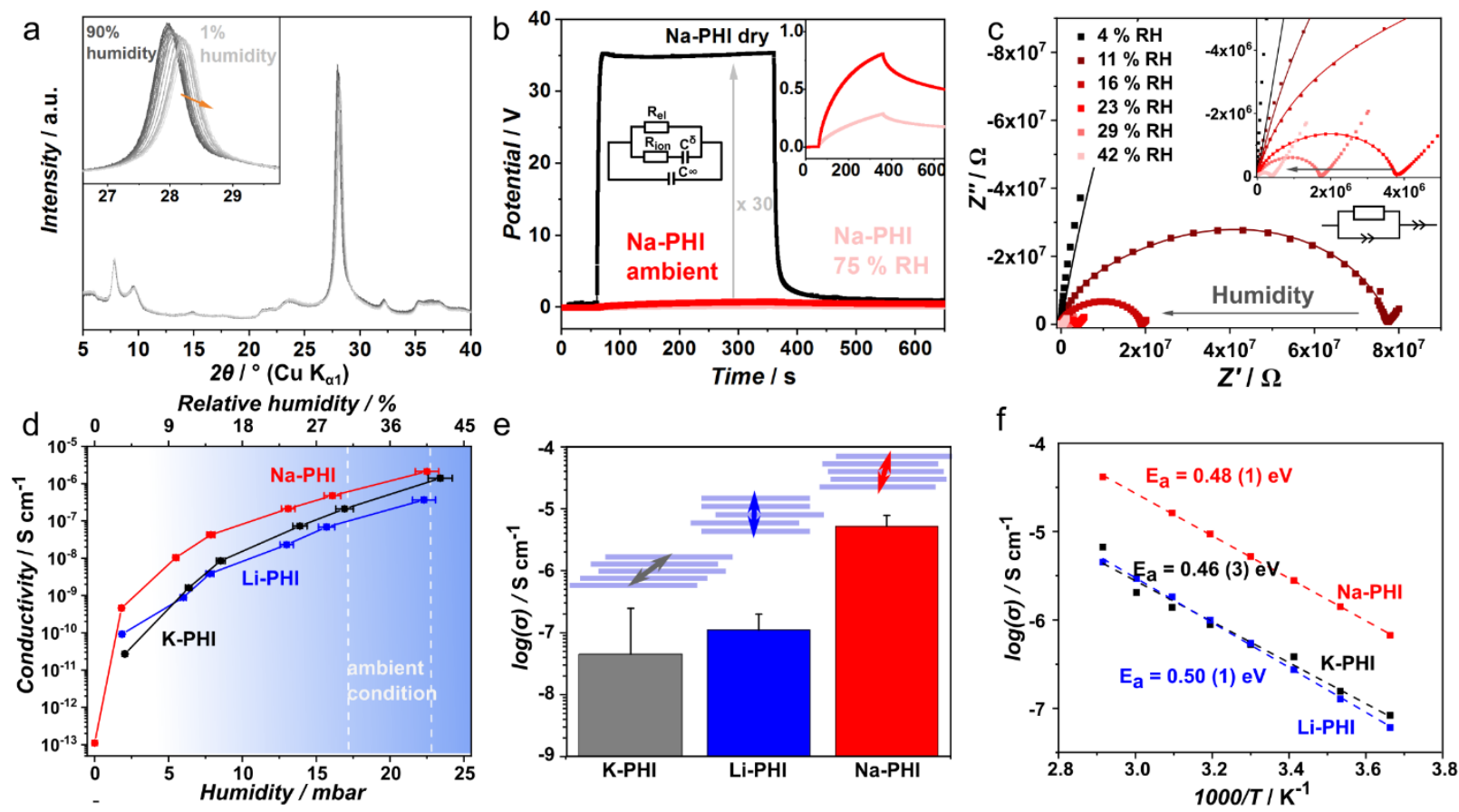

Figure 2: Analysis of ion conductivity in $M$-PHI. a) Humidity-dependent PXRD measurements of K-PHI from 1 to $90 \%$ RH in 5 $\%$ RH steps and the $1 \%$ RH steps for the last 5\%; b) DC polarization experiments of Na-PHI for a dry sample (dried in vaccum oven for 1 day) (black), ambient conditions (around $35 \% \mathrm{RH}$ ) (red) and $75 \% \mathrm{RH}$ (pink) with 2 nA applied; c) Humidity-dependent impedance measurements of Na-PHI from 4 up to $42 \%$ RH mbar at $308 \mathrm{~K}$ (Ru sputtered pellet, which did not change the resistance compared to steel electrodes (Table S10). Data of the first semi-circle were fitted with a (RQ) element; d) Overview of humiditydependent conductivity extracted from impedance spectroscopy of K-, Na- and Li-PHI at $308 \mathrm{~K}$ with Ru sputtered contacts; e) Comparison of overall conductivities estimated by impedance measurments of ambient K-, Na-, and Li-PHI with suggested 1D ion conduction along the pore channels; f) Arrhenius plot of K-, Na-, and Li-PHI to determine the activation energy of ion conductivity under ambient conditions.

An important question to address is whether the observed ionic conductivity is partially due to mobile protons, as described for melon-type carbon nitrides. ${ }^{[21,54]}$ To this end, the potassium ions were replaced by protons (forming H-PHI), and the conductivity was probed by EIS and DC measurements, which resulted in a two to three orders of magnitude lower overall conductivity $\left(10^{-9} \mathrm{~S} \mathrm{~cm}^{-1}\right)$ of H-PHI compared to $M$-PHI (Figure S41, S42, Supporting information), even though the water content is increased in H-PHI (Figure S17, Supporting information). For H-PHI a similar electronic conductivity as $M$-PHI $\left(10^{-9} \mathrm{~S} \mathrm{~cm}^{-1}\right)$ and an ionic conductivity of $10^{-10} \mathrm{~S} \mathrm{~cm}^{-1}$ was observed (Figure S27, Table S7, Supporting information). This suggests that the protons interact strongly with the PHI backbone and are not very mobile. At the same time, the even stronger conductivity decrease in Ba-PHI vs H-PHI is probably caused by structure disruption, 
hindering electron transport. We previously found electronic transport in PHI to be best described by a multiple trapping and release (MRT) process. ${ }^{[12,55]}$

Moreover, solid-state NMR spectroscopy was used to gain further insights into the microscopic mechanism of ion diffusion in Li- and Na-PHI. For all conditions both ${ }^{7} \mathrm{Li}$ and ${ }^{23} \mathrm{Na} \mathrm{NMR}$ exhibit one single signal, resulting from hydrated alkali ions in the pores. However, interactions between the backbone and the ions cannot be entirely ruled out, caused by signal broadening (Figure 2e, Figure S47, Supporting information). ${ }^{1} \mathrm{H}$ NMR analysis revealed a only broad signal around $4 \mathrm{ppm}$ caused by water in the PHI pores, which could not be removed completely, even after extended sample drying (Figure S48, Supporting information), thus precluding NMR analysis.

The activation energy obtained by temperature-dependent ${ }^{7} \mathrm{Li}$ PFG NMR in Li-PHI $\left(\mathrm{E}_{\mathrm{a}}=0.56\right.$ $( \pm 0.05) \mathrm{eV})$ (Figure S44, Supporting information) under ambient conditions (around $35 \% \mathrm{RH})$ is comparable to the values received from EIS. However, the determined tracer diffusion coefficients for the lithium ion motion are at the sensitivity limit of NMR, so that those values are just taken as an upper limit for the ionic diffusivity $\left(D^{\operatorname{tr}} 1.2( \pm 0.1) \times 10^{-13} \mathrm{~m}^{2} \mathrm{~s}^{-1}\right.$ at $343 \mathrm{~K}$ (Table S13, Supporting information), corresponding to $\left.1.3( \pm 0.2) \times 10^{-5} \mathrm{~S} \mathrm{~cm}^{-1}\right)$. When increasing the humidity to $75 \% \mathrm{RH}$, we revealed two diffusion processes above $353 \mathrm{~K}$, characterized by substantially different diffusion coefficients on the order of $10^{-12} \mathrm{~m}^{2} \mathrm{~s}^{-1}$ and $10^{-10} \mathrm{~m}^{2} \mathrm{~s}^{-1}$ (Figure S44, Supporting information). This observation highlights two different transport processes co-existing within the sample at higher humidity and particularly at elevated temperatures. The mechanism behind a faster ion motion at higher temperatures requires further studies. A reason might be an additional lithium motion scheme in confined spaces, textural voids, grain boundaries or at the particle surface due to multilayer water adsorption ${ }^{[56]}$ in contrast to the ion motion inside the pores, which are more hindered due to interaction of the ion (or its hydration shell) with the backbone, resulting in a small change of the diffusion coefficient with temperature.

The differences between the line width in dry, ambient (approximately $35 \% \mathrm{RH}$ ), and $75 \% \mathrm{RH} \mathrm{Li}$ PHI at room temperature $(303 \mathrm{~K})$ in ${ }^{7} \mathrm{Li} \mathrm{NMR}$ line broadening experiments further proves an enhancement of lithium mobility with increasing humidity (FWHM of $2838 \mathrm{~Hz}$ at dry samples vs. $1418 \mathrm{~Hz}$ under ambient conditions, $1044 \mathrm{~Hz}$ in $75 \% \mathrm{RH}$ ) (Figure 3a, Figure S47, Supporting information). Such a water-assisted increase in ion conduction has not only been shown for polymers ${ }^{[53,57]}$ but also for layered inorganic systems, ${ }^{[9]}$ highlighting that $2 \mathrm{D}$ carbon nitrides may 
be intriguing candidates for ion-conducting membranes or separators. However, it must be stated that a considerable contribution of proton motion, especially at high humidity values, could not be fully excluded to contribute to the overall conductivity of $M$-PHI.

Next, variation of static ${ }^{7} \mathrm{Li}$ and ${ }^{23} \mathrm{Na}$ NMR line shapes under ambient conditions was analyzed at different temperatures, and the onset of motional narrowing was estimated, which occurs when the $\mathrm{Li}$ or $\mathrm{Na}$ jump rate exceeds the rigid lattice line width, averaging out the local dipolar interaction between the Li or Na spins (Figure 3b,c, Figure S47-S49, Supporting information). The ${ }^{7} \mathrm{Li}$ signal is broad (full width at half maximum $(\mathrm{FWHM})=6700 \mathrm{~Hz})$ at $193 \mathrm{~K}$ and gradually narrows with increasing temperature to a FWHM of $604 \mathrm{~Hz}$ (at $373 \mathrm{~K}$ ), resulting in a jump rate of lithium of $\tau_{C}^{-1} \approx 6.7( \pm 5.0) \times 10^{3} \mathrm{~s}^{-1}$ at $229 \mathrm{~K}$ and an uncorrelated tracer diffusion coefficient $\left(\mathrm{D}^{\mathrm{uc}}\right)$ of 3.4 $( \pm 0.3) \times 10^{-16} \mathrm{~m}^{2} \mathrm{~s}^{-1}$ (Table S14, Supporting information). Extrapolating the conductivity values obtained by EIS, a smaller diffusion coefficient from EIS $\left(D^{\sigma} \sim 7 \times 10^{-17} \mathrm{~m}^{2} \mathrm{~s}^{-1}\right.$ at $\left.229 \mathrm{~K}\right)$ is obtained at similar temperatures, pointing to a faster ion motion on the microscopic scale analyzed in the FWHM analysis (on the $\mathrm{kHz}$ domain) in comparison to the macroscopic DC measurements. This is in line with a reduced activation energy of $0.37( \pm 0.01) \mathrm{eV}$ for the Li diffusion on a shorter time scale (Figure 3c) in contrast to $0.50( \pm 0.01) \mathrm{eV}$ for longer timescales. Further evidence of hindered lithium diffusion on the long-range due to the hydration shell can be found by humidity-dependent spin-lattice relaxation experiments for Li-PHI, which are sensitive to even shorter time intervals (Figure S46, Supporting information). In comparison, Na-PHI shows a slightly lower onset temperature and a jump rate of $\tau_{C}^{-1} \approx 7.1( \pm 6.0) \times 10^{3} \mathrm{~s}^{-1}$ at $206 \mathrm{~K}\left(\mathrm{D}^{\mathrm{uc}}=3.7( \pm 0.4) \times 10^{-16} \mathrm{~m}^{2} \mathrm{~s}^{-1}\right)$ (Figure S49, Supporting information), as well as a slightly lower activation energy of $0.33 \mathrm{eV}$ for shorter timescale measurements. This highlights a higher sodium mobility in the pores, also at shorter time scales in agreement to EIS results and substantiates the influence of the ion size and pore geometry on the mobility. However, at such low temperatures where the onset of motion narrowing is observed $(240 \mathrm{~K})$, water in the PHI pores might be frozen already, which would result in different activation energies and diffusion values, as evidenced by $\mathrm{T}_{1}$ experiments of ${ }^{7} \mathrm{Li},{ }^{23} \mathrm{Na}$, and ${ }^{1} \mathrm{H}$ below $240 \mathrm{~K}$ (Figure S45, S46, Supporting information). ${ }^{[58,59]}$ 

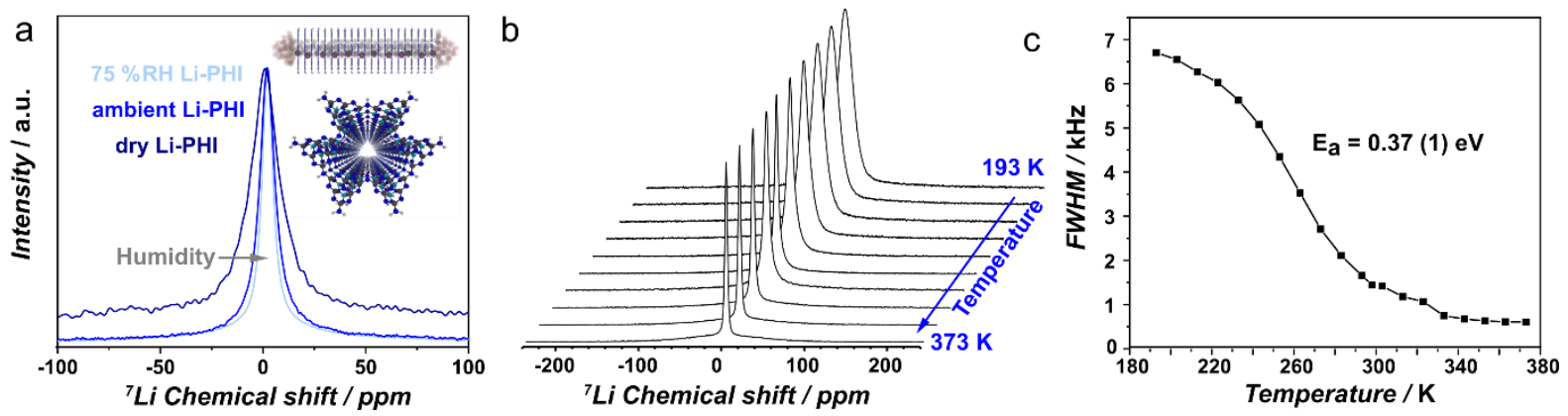

Figure 3: Humidity-dependent ${ }^{7} \mathrm{Li} \mathrm{NMR}$ measurments of Li-PHI. a) ${ }^{7} \mathrm{Li}$ NMR static measurments at $303 \mathrm{~K}$ of dry, ambient (approximatly $35 \% \mathrm{RH}$ ) and $75 \% \mathrm{RH}$ humid sample of Li-PHI. Inset shows the PHI pore filled with water and hydated ions from the side and an empty staggered stacked pore form the top; b) Variable temperature ${ }^{7} \mathrm{Li}$ ssNMR spectra of Li-PHI; c) Motional narrowing curve of the static ${ }^{7} \mathrm{Li}-\mathrm{NMR}$ line width (full width of half maximum (FWHM)) plotted against the temperature, with the corresponding activation energy for Li motion.

\section{Quantum chemical calculations}

To obtain further insights into the impact of the type of ion in the pores, the stacking and pore structure on the conductivity, quantum-chemical calculations were performed on a model system of 21 stacked PHI pores (Chapter 6, Supporting information). For Li-PHI, lithium ions were calculated to have a first hydration shell of 5 water molecules in the PHI pores (Figure S53, Supporting information), in accordance with free solvated lithium ions, which have a hydration shell of 4 or 6 water molecules according to literature. ${ }^{[32]}$ In Na- and K-PHI, the first hydration shell is reduced by one(for $\mathrm{Na}^{+}$) or two (for $\mathrm{K}^{+}$) water molecules for ions residing in the PHI pore channel, in contrast to free solvated ions with six $\left(\mathrm{Na}^{+}\right)$or seven $\left(\mathrm{K}^{+}\right)$water molecules, in agreement with previously published work on K-PHI (Figure S53, Supporting information). ${ }^{[4]}$ Energy calculations of the ion-water interaction energy in the PHI pores also reflect this trend of a more reduced hydration shell in PHI pores containing larger alkali ions. Here, potassium ions are found to have a slightly stronger interaction with the PHI pore structure than $\mathrm{Li}^{+}$and $\mathrm{Na}^{+}$, which might explain the lower conductivity of K-PHI vs. Na-PHI (Figure S54-S56, Supporting information) due to stronger binding of $\mathrm{K}^{+}$. The comparably high hydration energy of $\mathrm{Li}^{+}$ combined with the interaction energies of water molecules with the PHI backbone might explain the lower conductivity of $\mathrm{Li}^{+}$in comparison to $\mathrm{Na}^{+}$, resulting in an optimal ion-water-PHI polymer interaction for Na-PHI. Those results are in good agreement with the experimentally observed conductivity values. The theoretical results of two different pore model systems with different 
deprotonation pattern of the imide bridges (which however do not include stacking faults) strongly suggest that the charge distribution on the backbone along with the stacking behavior impacts ion diffusion measured in the experiment as well. Hence, an interplay of hydrated ion size, stacking order, and interaction strength of ions with the polymer backbone has to be considered together for their combined impact on cation conductivity.

\section{Correlation between conductivity and photocatalysis}

Having established that $M$-PHI materials show predominant ionic conductivity under humid conditions, we now turn to whether a correlation between the observed conductivity and the photocatalytic activities in these materials exists. ${ }^{[60,61]}$ One hint on a possible correlation was published recently, showing a more pronounced photoelectric response of K-PHI in the presence of a hole conducting polymer in contrast to bare K-PHI. ${ }^{[62]}$ Here, we use the series of $M$-PHI as a pertinent model system to study such correlations for the photocatalytic hydrogen evolution rate (HER). In addition, the optical bandgap is similar in all materials $(\sim 2.75 \mathrm{eV})$, besides Cs-, TMAand $\mathrm{NH}_{4}-\mathrm{PHI}$ with a slightly enlarged bandgap of $2.80 \mathrm{eV}$, probably caused by partial protonation (Figure S57, Supporting information). ${ }^{[4]}$

PHI is known to form long-lived electrons on the polymer backbone when illuminated in the absence of oxygen and in the presence of an electron donor, which quenches the light-induced holes. ${ }^{[5,6]}$ During this process, PHI itself remains stable (Figure S60, S61, Table S17, Supporting information). The charge accumulation on PHI is accompanied by a color change from yellow to blue, which allows for time delayed hydrogen evolution ${ }^{[5]}$ or other photocatalytic reactions, ${ }^{[7,63]}$ as well as solar battery applications, and is most probably the reason for an enhanced photocatalytic activity when compared to melon. To test the impact of ions in the charge stabilization, an aqueous suspension of H-PHI, without the mobile counterions, was illuminated in the presence of an electron donor, but no color change could be observed. However, when adding salts containing $\mathrm{Li}^{+}, \mathrm{Na}^{+}, \mathrm{K}^{+}, \mathrm{Cs}^{+}$or $\mathrm{NH}_{4}^{+}$, which increase the conductivity as discussed above, the suspension turns blue, highlighting the participation of ions in this process (Table S16, Supporting information). Those ions have to be mobile and fit into the pore, as demonstrated by the absence of the color change characteristic of photocharging when $\mathrm{Ba}^{2+}$ or $\mathrm{TMA}^{+}$salts were added. As such, it appears that mobile alkali ions reorganize upon light excitation to screen the trapped electrons 
on the PHI backbone (Figure 4c). A related process was described for 1D carbon nitride melon, which was shown to act as a light-driven ion pump. ${ }^{[64]}$ Upon asymmetric illumination, charges are generated at the surface, separated by electrons moving away from the illumination spot, which was ascribed to an n-type conductivity-induced band bending. This generated charge gradient was described as the driving force for the alkali ions in solution to follow the electrons. ${ }^{[64-66]}$
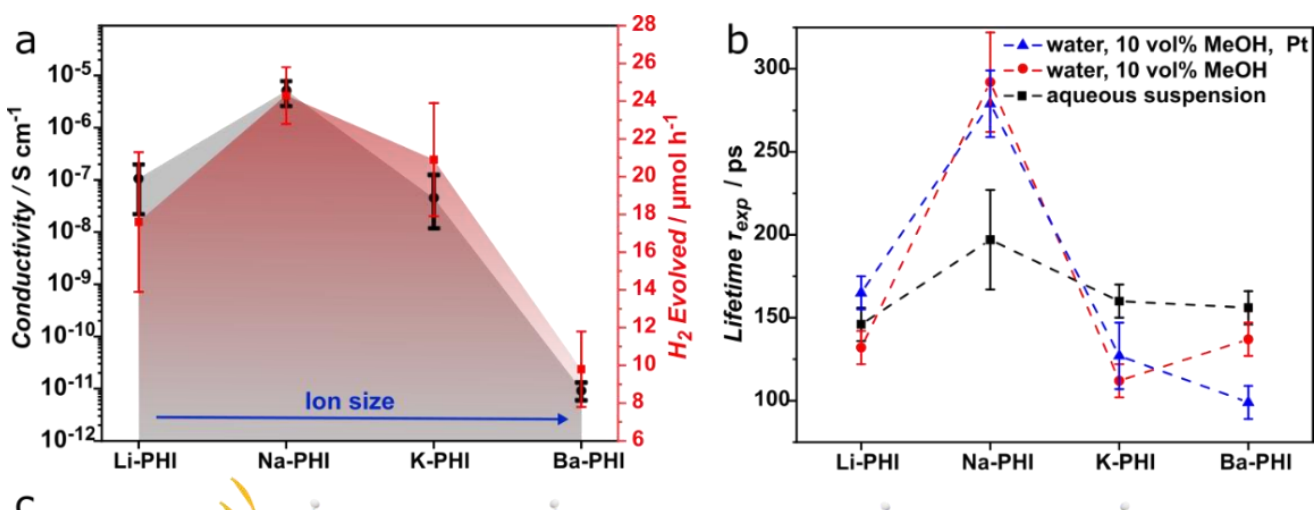

C
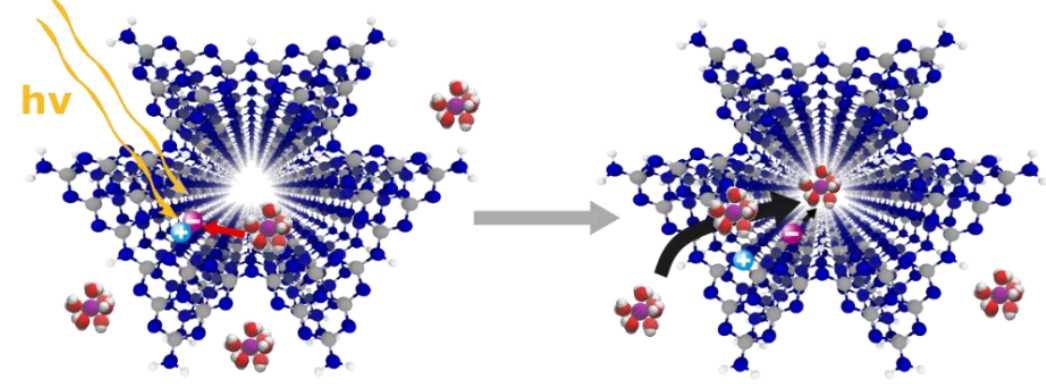

Figure 4: Optical characerisation and photocatalytic hydrogen evolution of K-, Na-, Li- and Ba-PHI. a) Hydrogen evolution of $M$ $\mathrm{PHI}$ at $100 \mathrm{~mW} \mathrm{~cm}^{-1}$ illumination in the presence of $10 \mathrm{vol} \% \mathrm{MeOH}$ and $8 \mathrm{wt} \% \mathrm{Pt}$ loading, and its correlation with the observed conductivity, measured for PHI pellets; b) Lifetimes of $M$-PHI detemined by time-dependent PL measurements in aqueous suspensions, aqueous suspensions with $10 \mathrm{vol} \% \mathrm{MeOH}$, and after photodeposition of Pt. Lifetime as fitted with two different functions represented by $\tau_{\exp }$ and $\tau_{\text {mode }}$; c) Scheme of photo-generated charges in $M$-PHI, which are stabilized by mobile alkali ions.

In photocatalysis experiments with suspended particles of similar size (500-1000 nm) we hypothesize that the mobile cations in the pores of PHI act to stabilize the trapped electrons through Coulombic interactions, while the pore water assists in dielectric screening and acts as a vehicle to facilitate movement of the cations through the pores additionally. As outlined above, the ionic conductivity is enhanced in humid environment and is therefore assumed to be the highest in an aqueous environment, approximating $100 \% \mathrm{RH}$, as applicable during photocatalysis in aqueous suspension. Indeed, in line with the conductivity results, we observe the highest photocatalytic HER activity for Na-PHI $\left(24( \pm 1) \mu \mathrm{mol} \mathrm{h}^{-1}\right)$ compared to Li- and K-PHI $\left(18( \pm 4) \mu \mathrm{mol} \mathrm{h}{ }^{-1}\right.$ and 19 $\left.( \pm 4) \mu \mathrm{mol} \mathrm{h}^{-1}\right)$, under illumination with simulated sunlight and in the presence of $8 \mathrm{wt} \% \mathrm{Pt}$ loading 
acting as co-catalyst (Figure 4a). When comparing the photocatalytic activity and the conductivity, a similar correlation was identified for $M$-PHI with the other cations studied (Figure S58, S59, Supporting information), e.g. the lowest photocatalytic activity results for Ba-PHI $(10( \pm 1) \mu$ mol $\mathrm{h}^{-1}$ ), which also has the lowest overall conductivity. We rationalize the observed trends in photocatalysis and color change experiments by a more efficient stabilization of the photogenerated charges by ions being mobile in the structure. Nevertheless, it must be stated that an excessive stabilization of charges on the material can also have detrimental effects on the photocatalytic activity, since excessively accumulated, photo-generated charges, cannot be used for the redox reactions anymore and can recombine. ${ }^{[8]}$ This is resulting in a sweet spot of charge stabilization and photocatalytic efficiency, where the time scales of charge stabilization and photocatalysis are suitably matched.

To further substantiate this behavior in PHI, where mobile alkali ions are already built into the system as opposed to melon, electrochemical measurements in the presence of light and photoluminescence (PL) studies were performed. PL emission and lifetime measurements in aqueous suspension of PHI (containing, $\mathrm{Li}^{+}, \mathrm{Na}^{+}, \mathrm{K}^{+}, \mathrm{Ba}^{2+}$ ) in the presence and absence of methanol as well as after Pt deposition were performed systematically to study the effect of each component used for photocatalysis experiments. The PL emission spectra of all alkali ion-bearing PHI materials is similar, with a maximum intensity around $450 \mathrm{~nm}$ (Figure S62, S63, Supporting information). However, the relative PL intensity is not a suitable measure for the photocatalytic performance due to the overall low PL quantum yields observed for PHI.

Time-resolved PL measurements, excited at a wavelength of $370 \mathrm{~nm}$, were fitted by an exponential decay function and a $\Gamma$ distribution (Figure S64, Supporting information) ${ }^{[67,68]}$ highlighting at least two different decay processes being present on the probed time scales in the material. The shorter lifetime $\left(\tau_{\exp }\right.$ ) of $\sim 110$ ps up to $\sim 290$ ps corresponds to an internal recombination of charge carriers in the bulk of PHI, whereas the longer lifetime ( $\tau_{\text {mode }}$ ) of 200 ps to 840 ps is likely caused by decay processes on the surface (Table S18, Supporting information). ${ }^{[8,12,69]}$ When comparing the lifetime corresponding to the bulk properties in different environments, it can be clearly seen that lifetimes of Na-PHI are prolonged by $20-60 \%$ in comparison to the other $M$-PHI materials, which corresponds to a lower overall recombination rate in the Na-PHI bulk (Figure 4b). This behavior is consistent with the assumption of ion-assisted stabilization of light-induced electrons. Prolonged 
lifetimes of the photo-induced charge carriers in turn increase the probability of productively using the generated electrons in the photocatalytic HER, which is in line with the highest photocatalytic activity observed for Na-PHI. To estimate the impact of ion motion on possible charge stabilization, a diffusion length $(\mathrm{L}=\sqrt{D * \tau})$ of the ions in the pores can be calculated on the time scales extracted from PL with the conductivity diffusion coefficients $\left(\mathrm{D}^{\sigma}\right)$ determined above for humid pellets (Table S15, Supporting information). ${ }^{[70]}$ Diffusion lengths of 0.10 (for K-and LiPHI) to $0.35 \AA$ (for Na-PHI) were calculated, which will be significantly higher under aqueous conditions, but presumably with a preserved relative trend (Figure 3c). This however means that within the measured timescales, only short range motion is enabled, which is likely sufficient to reach and stabilize an electron residing nearby (polaronic effect), ${ }^{[65]}$ and hence is most efficient for Na-PHI with the highest ion conductivity. A diffusion distance corresponding to the layer separation (3.2 $\AA$ ) would require for example roughly $22 \mathrm{~ns}$ for $\mathrm{Na}-\mathrm{PHI}$, which is too long to be detected by PL, since the radiative decay signal being used as measure for the charge recombination process is shorter lived. Nevertheless, the PL data shows a contribution of ion stabilizing the photo-generated charge already on short time and length scales, which is described to be most important for an efficient charge separation. ${ }^{[71]}$

To probe the possible effect of ion-mediated charge stabilization on the photocatalytic activity on the timescale of long-lived electrons, which are observable by the blue state, the build-up of a photopotential and its relaxation in $M$-PHI pellets under ambient conditions during illumination with AM $1.5 \mathrm{G}\left(100 \mathrm{~mW} \mathrm{~cm}^{-1}\right)$ was studied (Figure S65, Supporting information). It can be seen that a negative photovoltage builds up instantaneously on the illumination side in all cases. After illumination, the generated photopotential decays, most slowly for Na-PHI while not reaching the initial potential after 30s in the dark. This behavior signifies that the photoexcited electrons are stabilized on the material (negative shift in Fermi energy), probably due to charge trapping assisted by efficient $\mathrm{Na}^{+}$migration. For the other $M$-PHI materials, the negative photovoltage decay occurs faster, which points to a less efficient charge stabilization after photoexcitation. This trend, showing clear differences in magnitude and time scale of charge stabilization, is in line with the findings from conductivity and PL lifetime measurements, and suggests a correlation between conductivity, electrostatic screening and charge carrier lifetimes. Since these factors are consistent with trend observed for the photocatalytic HER activity of $M$-PHI, our study points to a correlation between ion conductivity, charge stabilization and photocatalytic efficiency. Although a causal 
relationship between these factors can neither be proven nor excluded at this stage, we emphasize that enhancing the conductivity of a material may be an important, yet so far largely overlooked design parameter to enhance the photocatalytic activity. If conductivity -ionic or electronic - is limiting photocatalysis, it can cause significant recombination and be detrimental for charge extraction, since in that case only those charge carriers generated and separated close to the solidliquid interphase remain available for photocatalytic reactions. ${ }^{[10]}$

\section{Conclusion}

In this study, the impact of ion mobility in the structural pores of 2D carbon nitride poly(heptazine imide) and its influence on the stabilization of photo-generated charges and hence, the photocatalytic hydrogen evolution was investigated. First, $M$-PHI $\left(M=\mathrm{K}^{+}, \mathrm{Li}^{+}, \mathrm{Cs}^{+}, \mathrm{NH}_{4}^{+}, \mathrm{TMA}^{+}\right.$, $\mathrm{Ba}^{2+}$ ) was structurally analyzed with different counterions being present in the pores. Depending on the pore content and the size of the hydrated cations, the stacking of the 2D layers and thereby the pore geometry is modified while the polymer backbone remains unchanged. The differences between effective channel sizes, hydrodynamic cation radius and ion-backbone interactions for the different $M$-PHI translate into distinctly different ion motion within the PHI pore channels. Ion transport is found to be fastest for Na-PHI, which outperforms the other cation containing PHI materials by minimum one order of magnitude. Irrespective of the cation, the conductivity of the ions increases significantly with the water content, which influences the electrostatic interactions between the ions and the polymer backbone. For Na-PHI, which showed the fastest ion motion under all conditions, the conductivity is increased by 5 to 6 orders of magnitude (from $10^{-9} \mathrm{~S} \mathrm{~cm}^{-1}$ to $10^{-4} \mathrm{~S} \mathrm{~cm}^{-1}$ ) with increasing relative humidity from 2 to around $80 \%$.

With the documented ability of PHI to stabilize and trap photo-generated electrons over hours, we studied the influence of the mobile ions in $M$-PHI on the charge trapping ability on short time scales and on the photocatalytic hydrogen evolution activity of PHI. A fast cation motion was found to be beneficial for increasing the photo-generated charge carrier lifetimes in PHI on short (ps) and long (s) time scales, which shows a positive correlation with the hydrogen evolution rate,

likely caused by the interaction between the photo-generated electron and the mobile hydrated cations in the PHI pores. In agreement with the ionic conductivity values, the longest PL lifetimes were found for Na-PHI, which also exhibits the highest hydrogen evolution rate. For Na-PHI, the size of the hydrated cation shows an optimal match with the effective PHI channel size, which is 
determined by finite, but small random layer shifts that enable high mobility of the hydrated $\mathrm{Na}$ ions in the pores.

From our findings we conclude that the ionic conductivity is an important, but so far underappreciated design parameter in catalyst design, especially in porous materials or materials where the conductivity can represent a bottleneck for immediate charge carrier stabilization and extraction. And yet, charge stabilization by motion of the mobile counterion in porous organic semiconductors is not only relevant for photocatalysis, but also for the realization of field effect transistors, chemical capacitors or sensors, where a high sensitivity for water and ion content is desirable to tune the material properties and thus, the read-out/output, such as conductivity.

\section{Acknowledgements}

Financial support by the Max Planck Society, the ERC Starting Grant (project COFLeaf, grant number 639233), the Center for NanoScience, DFG cluster of excellence "e-conversion" (EXC 2089/1-390776260) is gratefully acknowledged. C.O. acknowledges additional financial support as a Max Planck Fellow at the Max Planck Institute for Solid State Research, Stuttgart. A.J.S. gratefully acknowledges a postdoctoral scholarship from the Max Planck Society. The authors thank M.-L- Schreiber for elemental analysis and N. Vargas-Barbosa for fruitful discussions. The authors acknowledge DESY (Hamburg, Germany), a member of the Helmholtz Association HGF,

for the provision of experimental facilities. Parts of this research were carried out at beamline P21.1, and Soham Banerjee is thanked for performing total scattering measurements.

\section{References}

[1] B. S. R. o. W. Energy, Statistical review of world energy, Vol. 69, BP, 2020.

[2] X. Wang, K. Maeda, A. Thomas, K. Takanabe, G. Xin, J. M. Carlsson, K. Domen, M. Antonietti, Nat. Mater. 2009, 8 (1), 76-80.

[3] R. Malik, V. K. Tomer, Renewable and Sustainable Energy Reviews 2021, 135, 110235.

[4] H. Schlomberg, J. Kröger, G. Savasci, M. W. Terban, S. Bette, I. Moudrakovski, V. Duppel, F. Podjaski, R. Siegel, J. Senker, R. E. Dinnebier, C. Ochsenfeld, B. V. Lotsch, Chem. Mater. 2019, 31 (18), 7478-7486.

[5] V. W.-h. Lau, D. Klose, H. Kasap, F. Podjaski, M.-C. Pignié, E. Reisner, G. Jeschke, B. V. Lotsch, Angew. Chem. Int. Ed. 2017, 56 (2), 510-514.

[6] F. Podjaski, J. Kröger, B. V. Lotsch, Adv. Mater. 2018, 30 (9), 1705477.

[7] V. Sridhar, F. Podjaski, J. Kröger, A. Jiménez-Solano, B.-W. Park, B. V. Lotsch, M. Sitti, Proc. Natl. Acad. Sci. U. S. A. 2020, 117 (40), 24748-24756.

[8] W. Yang, R. Godin, H. Kasap, B. Moss, Y. Dong, S. A. J. Hillman, L. Steier, E. Reisner, J. R. Durrant, J. Am. Chem. Soc. 2019, 141 (28), 11219-11229. 
[9] V. Augustyn, Y. Gogotsi, Joule 2017, 1 (3), 443-452.

[10] T. Banerjee, F. Podjaski, J. Kröger, B. P. Biswal, B. V. Lotsch, Nat. Rev. Mater. 2021, 6 (2), 168190.

[11] K. Takanabe, ACS Catal. 2017, 7 (11), 8006-8022.

[12] J. Kröger, A. Jiménez-Solano, G. Savasci, V. W. h. Lau, V. Duppel, I. Moudrakovski, K. Küster, T. Scholz, A. Gouder, M.-L. Schreiber, F. Podjaski, C. Ochsenfeld, B. V. Lotsch, Adv. Funct. Mater. 2021, 31 (28), 2102468.

[13] X. Li, A. F. Masters, T. Maschmeyer, ChemCatChem 2015, 7 (1), 121-126.

[14] C. Merschjann, T. Tyborski, S. Orthmann, F. Yang, K. Schwarzburg, M. Lublow, M. C. LuxSteiner, T. Schedel-Niedrig, Phys. Rev. B 2013, 87 (20), 205204.

[15] C. Merschjann, S. Tschierlei, T. Tyborski, K. Kailasam, S. Orthmann, D. Hollmann, T. SchedelNiedrig, A. Thomas, S. Lochbrunner, Adv. Mater. 2015, 27 (48), 7993-7999.

[16] M. N. Huda, J. A. Turner, J. Appl. Phys. 2010, 107 (12), 123703.

[17] Y. Noda, C. Merschjann, J. Tarábek, P. Amsalem, N. Koch, M. J. Bojdys, Angew. Chem. Int. Ed. 2019, 58 (28), 9394-9398.

[18] K. A. See, S. Hug, K. Schwinghammer, M. A. Lumley, Y. Zheng, J. M. Nolt, G. D. Stucky, F. Wudl, B. V. Lotsch, R. Seshadri, Chem. Mater. 2015, 27 (11), 3821-3829.

[19] Z. Zhang, J. Huang, Q. Yuan, B. Dong, Nanoscale 2014, 6 (15), 9250-9256.

[20] R. Malik, V. K. Tomer, V. Chaudhary, M. S. Dahiya, A. Sharma, S. P. Nehra, S. Duhan, K. Kailasam, J. Mater. Chem. A 2017, 5 (27), 14134-14143.

[21] V. K. Tomer, N. Thangaraj, S. Gahlot, K. Kailasam, Nanoscale 2016, 8 (47), 19794-19803.

[22] S. P. Lee, J. G. Lee, S. Chowdhury, Sensors 2008, 8 (4), 2662-2672.

[23] H. Arazoe, D. Miyajima, K. Akaike, F. Araoka, E. Sato, T. Hikima, M. Kawamoto, T. Aida, Nat. Mater. 2016, 15 (10), 1084-1089.

[24] S. Cao, J. Low, J. Yu, M. Jaroniec, Adv. Mater. 2015, 27 (13), 2150-2176.

[25] L. Ge, F. Zuo, J. Liu, Q. Ma, C. Wang, D. Sun, L. Bartels, P. Feng, J. Phys. Chem. C 2012, 116 (25), 13708-13714.

[26] S. Gope, S. Malunavar, A. J. Bhattacharyya, ChemistrySelect 2018, 3 (19), 5364-5376.

[27] V. W.-h. Lau, I. Moudrakovski, T. Botari, S. Weinberger, M. B. Mesch, V. Duppel, J. Senker, V. Blum, B. V. Lotsch, Nat. Commun. 2016, 7 (1), 12165.

[28] A. Savateev, S. Pronkin, M. G. Willinger, M. Antonietti, D. Dontsova, Chem.-Asian J. 2017, 12 (13), 1517-1522.

[29] A. Coelho, J. Appl. Crystallogr. 2018, 51 (1), 210-218.

[30] A. M. Pütz, M. W. Terban, S. Bette, F. Haase, R. E. Dinnebier, B. V. Lotsch, Chem. Sci. 2020, 11 (47), 12647-12654.

[31] H. Y. Liu, Y. Q. Zhou, F. Y. Zhu, W. Q. Zhang, G. G. Wang, Z. F. Jing, C. H. Fang, Eur. Phys. J. D 2020, 74 (1), 2.

[32] J. Mähler, I. Persson, Inorg. Chem. 2012, 51 (1), 425-438.

[33] X. Wang, D. Toroz, S. Kim, S. L. Clegg, G.-S. Park, D. Di Tommaso, Phys. Chem. Chem. Phys. 2020, 22 (28), 16301-16313.

[34] X. Guo, S. Theissen, J. Claussen, V. Hildebrand, J. Kamphus, M. Wilhelm, B. Luy, G. Guthausen, Macromol. Chem. Phys. 2019, 220 (2), 1800350.

[35] G. A. Gerencser, Epithelial Transport Physiology, Humana Press, 2010.

[36] E. R. Nightingale, J. Phys. Chem. 1959, 63 (9), 1381-1387.

[37] S. Ringe, E. L. Clark, J. Resasco, A. Walton, B. Seger, A. T. Bell, K. Chan, Energ. Environ. Sci. 2019, 12 (10), 3001-3014.

[38] J. K. W. Chui, T. M. Fyles, Chem. Soc. Rev. 2012, 41 (1), 148-175.

[39] F. Guo, S. Li, Y. Hou, J. Xu, S. Lin, X. Wang, Chem. Commun. 2019, 55 (75), 11259-11262.

[40] P. Atkins, J. de Paula, Atkins' physical chemistry, Oxford University Press, New York, 2002.

[41] Y. Cao, L. Xiao, M. L. Sushko, W. Wang, B. Schwenzer, J. Xiao, Z. Nie, L. V. Saraf, Z. Yang, J. Liu, Nano Lett. 2012, 12 (7), 3783-3787. 
[42] A. Kuhn, S. Dupke, M. Kunze, S. Puravankara, T. Langer, R. Pöttgen, M. Winter, H.-D. Wiemhöfer, H. Eckert, P. Heitjans, J. Phys. Chem. C 2014, 118 (49), 28350-28360.

[43] V. Epp, Q. Ma, E.-M. Hammer, F. Tietz, M. Wilkening, Phys. Chem. Chem. Phys. 2015, 17 (48), 32115-32121.

[44] A. Kuhn, V. Duppel, B. V. Lotsch, Energ. Environ. Sci. 2013, 6 (12), 3548-3552.

[45] A. Senocrate, I. Moudrakovski, G. Y. Kim, T.-Y. Yang, G. Gregori, M. Grätzel, J. Maier, Angew. Chem. Int. Ed. 2017, 56 (27), 7755-7759.

[46] T.-Y. Yang, G. Gregori, N. Pellet, M. Grätzel, J. Maier, Angew. Chem. Int. Ed. 2015, 54 (27), 7905-7910.

[47] N. G. Park, M. Grätzel, T. Miyasaka, Organic-inorganic halide perovskite photovoltaics: From fundamentals to device architectures, 2016, 1-366.

[48] G. Y. Kim, A. Senocrate, T.-Y. Yang, G. Gregori, M. Grätzel, J. Maier, Nat. Mater. 2018, 17 (5), 445-449.

[49] D. Prutsch, B. Gadermaier, H. Brandstätter, V. Pregartner, B. Stanje, D. Wohlmuth, V. Epp, D. Rettenwander, I. Hanzu, H. M. R. Wilkening, Chem. Mater. 2018, 30 (21), 7575-7586.

[50] N. S. Schauser, G. E. Sanoja, J. M. Bartels, S. K. Jain, J. G. Hu, S. Han, L. M. Walker, M. E. Helgeson, R. Seshadri, R. A. Segalman, Chem. Mater. 2018, 30 (16), 5759-5769.

[51] Y. Hu, N. Dunlap, S. Wan, S. Lu, S. Huang, I. Sellinger, M. Ortiz, Y. Jin, S.-h. Lee, W. Zhang, J. Am. Chem. Soc. 2019, 141 (18), 7518-7525.

[52] D. M. DeLongchamp, P. T. Hammond, Chem. Mater. 2003, 15 (5), 1165-1173.

[53] M. Wieland, C. Dingler, R. Merkle, J. Maier, S. Ludwigs, ACS Appl. Mater. Inter. 2020, 12 (5), 6742-6751.

[54] J. Li, Y.-H. Yang, Q. Chen, W.-Q. Zhu, J.-H. Zhang, J. Mater. Chem. C 2020, 8 (12), 4065-4072.

[55] C. Adler, S. Selim, I. Krivtsov, C. Li, D. Mitoraj, B. Dietzek, J. R. Durrant, R. Beranek, Adv. Funct. Mater. n/a (n/a), 2105369.

[56] H. Park, P. P. M. Schleker, Z. Liu, N. Kowalew, T. Stamm, R. Schlögl, R.-A. Eichel, S. Heumann, J. Granwehr, J. Phys. Chem. C 2019, 123 (41), 25146-25156.

[57] M. Liu, P. Xia, L. Zhang, B. Cheng, J. Yu, ACS Sustain. Chem. Eng. 2018, 6 (8), 10472-10480.

[58] S. Nakhal, D. Wiedemann, B. Stanje, O. Dolotko, M. Wilkening, M. Lerch, J. Solid State Chem. 2016, 238, 60-67.

[59] A. Kuhn, M. Kunze, P. Sreeraj, H. D. Wiemhöfer, V. Thangadurai, M. Wilkening, P. Heitjans, Solid State Nucl. Magn. Reson. 2012, 42, 2-8.

[60] H. Gao, S. Yan, J. Wang, Y. A. Huang, P. Wang, Z. Li, Z. Zou, Phys. Chem. Chem. Phys. 2013, 15 (41), 18077-18084.

[61] S. Yang, Y. Gong, J. Zhang, L. Zhan, L. Ma, Z. Fang, R. Vajtai, X. Wang, P. M. Ajayan, Adv. Mater. 2013, 25 (17), 2452-2456.

[62] A. Savateev, Y. Markushyna, C. M. Schüßlbauer, T. Ullrich, D. M. Guldi, M. Antonietti, Angew. Chem. Int. Ed. 2021, 60 (13), 7436-7443.

[63] A. Savateev, B. Kurpil, A. Mishchenko, G. Zhang, M. Antonietti, Chem. Sci. 2018, 9 (14), 35843591.

[64] K. Xiao, L. Chen, R. Chen, T. Heil, S. D. C. Lemus, F. Fan, L. Wen, L. Jiang, M. Antonietti, Nat. Commun. 2019, 10 (1), 74.

[65] F. Podjaski, B. V. Lotsch, Adv. Energy Mater. 2021, 11 (4), 2003049.

[66] P. Jia, Q. Wen, D. Liu, M. Zhou, X. Jin, L. Ding, H. Dong, D. Lu, L. Jiang, W. Guo, Small 2019, 15 (50), 1905355.

[67] A. C. Fogarty, A. C. Jones, P. J. Camp, Phys. Chem. Chem. Phys. 2011, 13 (9), 3819-3830.

[68] J. Kröger, A. Jiménez-Solano, G. Savasci, P. Rovó, I. Moudrakovski, K. Küster, H. Schlomberg, H. A. Vignolo-González, V. Duppel, L. Grunenberg, C. B. Dayan, M. Sitti, F. Podjaski, C. Ochsenfeld, B. V. Lotsch, Adv. Energy Mater. 2021, 11 (6), 2003016.

[69] R. Godin, Y. Wang, M. A. Zwijnenburg, J. Tang, J. R. Durrant, J. Am. Chem. Soc. 2017, 139 (14), 5216-5224. 
[70] H. Mehrer, Diffusion in Solids: Fundamentals, Methods, Materials, Diffusion-Controlled Processes, Springer Berlin Heidelberg, 2007.

[71] S. Shoaee, M. Stolterfoht, D. Neher, Adv. Energy Mater. 2018, 8 (28), 1703355.

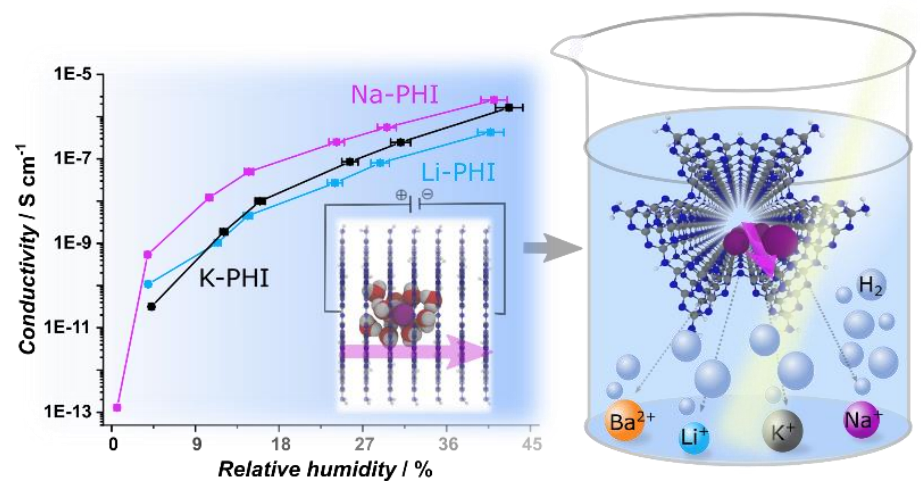

